\title{
G

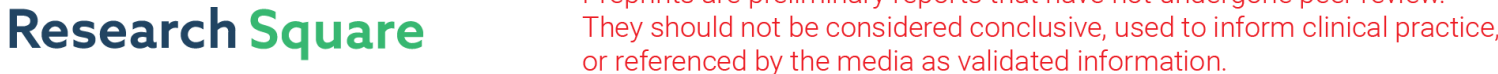 \\ Hemopneumothorax Detection Through the Process of Artificial Evolution - A Feasibility Study
}

\section{Adir Sommer ( $\nabla$ adirsommer@gmail.com )}

Israel Defense Forces https://orcid.org/0000-0003-3568-2489

\section{Noy Mark}

Israel Defense Forces

\section{Rafi Gerasi \\ Israel Defense Forces}

\section{Linn Wagnert Avraham}

Hebrew University Hadassah Medical School

\section{Ruth Fan-Marko}

Hebrew University of Jerusalem Faculty of Medicine

\section{Arik Eisenkraft}

Hebrew University of Jerusalem Faculty of Medicine

\section{Dean Nachman}

Hebrew University of Jerusalem Faculty of Medicine

\section{Research}

Keywords: Pneumothorax, Hemothorax, Trauma, Battlefield, Artificial evolution, Machine learning

Posted Date: October 29th, 2020

DOl: https://doi.org/10.21203/rs.3.rs-68799/v3

License: (c) (i) This work is licensed under a Creative Commons Attribution 4.0 International License. Read Full License

Version of Record: A version of this preprint was published at Military Medical Research on April 25th, 2021. See the published version at https://doi.org/10.1186/s40779-021-00319-2. 


\section{Abstract}

Background: Tension pneumothorax is one of the leading causes of preventable death on the battlefield. Current prehospital diagnosis relies on a subjective clinical impression complemented by a manual thoracic and respiratory examination. These techniques are not fully applicable in field conditions and on the battlefield, where situational and environmental factors may impair clinical capabilities. We aimed to assemble a device able to sample, analyze, and classify the unique acoustic signatures of pneumothorax and hemothorax.

Methods: Tested on an ex-vivo porcine model, we have assembled a device consisting of two sensitive digital stethoscopes and sampled 12 seconds of mechanical ventilation breathing sounds over the animals' thorax. The thoracic cavity was injected with increasing volumes of $200,400,600,800$, and $1000 \mathrm{ml}$ of air and saline to simulate pneumothorax and hemothorax, respectively. The data was analyzed using a multi-objective genetic algorithm that was used to develop an optimal mathematical detector through the process of artificial evolution, a cutting-edge approach in the artificial intelligence discipline.

Results: The algorithm was able to classify the signals according to their distinctive characteristics and to accurately predict, in up to $80 \%$ of cases, the presence of pneumothorax and hemothorax, starting from $400 \mathrm{ml}$, and regardless of background noise.

Conclusions: We present a potential objective and rapid diagnosis modality that can overcome independent and subjective factors that may delay diagnosis and treatment of potentially lethal thoracic injuries, with emphasis on field conditions. A future diagnostic device could be embedded with the algorithm and provide real-time detection and monitoring of pneumothorax and hemothorax.

\section{Background}

Traumatic pneumothorax, namely tension pneumothorax (tPTX) is one of the leading causes of preventable death on the battlefield [1-4]. These findings are consistent among researchers examining case fatality rates in various conflicts. tPTX was estimated to be the cause of up to $5 \%$ of preventable deaths among fatally wounded combat casualties in the Vietnam war [4]. Studies examining more recent conflicts exhibit similar findings, stating TPTX to be among the most common potentially survivable injuries [5-9]. Traumatic PTX may also be associated with hemothorax (HTX) [10,11], which is independently associated with an increased risk for mortality [8]. Although tPTX may be seen in approximately two percent of patients who suffer from simple pneumothorax, it is more likely to appear in trauma settings in light of poor diagnosis and treatment means [12].

Pre-hospital diagnosis of PTX and HTX is clinically made following a point of injury physical examination. In patients with a small PTX, findings on physical examination may be indistinct or may be limited until major clinical worsening has appeared. While various imaging tests, such as computerized tomography and chest x-ray can confirm the diagnosis, they are not employed in the pre-hospital setting. 
Emergency ultrasonography, namely the Extended Focused Assessment with Sonography for Trauma (EFAST) protocol, is being utilized by pre-hospital providers to evaluate for PTX in addition to HTX. Although most favorable studies present high overall diagnostic accuracy for PTX detection in major trauma $(>97 \%)[13,14]$, it is heavily relied on provider experience and therefore may be time consuming in the hands of a non-trained medical personnel.

Accuracy of physical examination in patients who present with penetrating or blunt chest trauma varies as it is highly subjective and depends on the physician's skills and experience. The sensitivity of auscultation was previously demonstrated to be as low as $50 \%$ in patients admitted with various degrees of hemopneumothorax due to penetrating or blunt chest trauma [15]. Likewise, Kong et al. presented that standard physical examination, consisting of an overall clinical impression, respiratory rate, oxygen saturation, tracheal deviation, chest expansion, percussion and auscultation findings, in a major trauma center, exhibited low sensitivity in diagnosing PTX and HTX (59\% [51-66, 95\% Cl] and 79\% [72-86, 95\% $\mathrm{Cl}$, respectively) [16]. Similar results for HTX, PTX, and various degrees of hemopneumothorax were also described by Chen et al. especially in patients with gunshot wounds with auscultation missing HTX in volume up to $600 \mathrm{ml}$, and hemopneumothorax up to $800 \mathrm{ml}$ [17]. Later, they demonstrated higher sensitivity rates, up to $84 \%$ in a prospective study conducted on 148 trauma patients [18]. As these studies were performed in a controlled quiet environment and under no time constraints, it is important to note that in field conditions, and specifically during combat, medical treatment is given under fire, in poor visibility, in the presence of intense background noise, under severe mental and physical stress, and under strict time limits. As such, physical examination may be extremely difficult to perform, and clinical impression may be vague, leading to a potentially slow and poor diagnosis as depicted in Figure 1 .

There are currently several pre-hospital non-invasive technological solutions that may help diagnose PTX and HTX. These include ultrasound- [19-21], radio waves- [22-24], and infrared thermography-based techniques [25]. Nevertheless, these techniques do not provide a robust solution and present numerous limitations such as specifically low detection rate in obese or muscular patients [19], difficulty in identifying the injured side [26], and a wide range of overall accuracy rate [23,24].

In our study, we present a prototype of a sampling device which utilizes a novel algorithm with a potential to non-invasively detect and quantify the presence of air and fluid in the plural cavity, independently of external factors that may delay diagnosis and treatment.

\section{Methods}

Type of study: An analytic experimental study in an ex-vivo porcine model.

Study design: The study was conducted in four white domestic female pigs (Laboratory Animals Farm, Lahav, Israel), aged 4 months (41-50 kg), housed in the institutional animal facility accredited by the Association for Assessment and Accreditation of Laboratory Animal Care International (AAALAC). Water and normal appropriate diet were available ad libitum. The experimental procedures were performed seven days after acclimatization. Food was withheld from the night before the procedure. Animals were 
sedated with Xylazine (1 mg/kg, IM, Eurovet Animal Health BV, The Netherlands) and anesthesia was induced with Ketamine (10 mg/kg, IM, Vetoquinol SA, Lure Cedex, France). The ear vein was then cannulated for intravenous administration of a mixture of Diazepam (2 mg, IV, TEVA Pharmaceutical Industries Ltd, Noida, India), Ketamine (400 mg, IV), Propofol (1-4 mg/kg, IV, Fresenius Kabi Austria Gmbh, Austria), and Tramadol (5 mg/kg, IM, Rafa Laboratories Ltd, Jerusalem, Israel) for analgesia. The pigs were then intubated with a cuffed silastic endotracheal tube (7.0-mm, Portex Tracheal Tube, UK). Anesthesia was maintained with $2 \%$ isoflurane (Piramal Critical Care Inc, Pa) in 100\% oxygen, and animals were ventilated using controlled mechanical ventilation (Excel 210-SE anesthesia machine Datex-Ohmeda Inc, Madison, Wis) or Narkomed-2B Anesthesia Machine - North American Drager, Pa). Tidal volume was set to $10 \mathrm{~mL} / \mathrm{kg}$ with respiratory rate of 13 to 15 breaths per minute adjusted to an endtidal $\mathrm{CO} 2$ (ETCO2) $35 \mathrm{~mm} \mathrm{Hg}$ at baseline. The animals were euthanized with an intravenous injection of $\mathrm{KCl}$ solution (Fagron Group BV, Rotterdam, The Netherlands). The porcine model was chosen due to its physiological and anatomical similarity to the human thorax including chest wall thickness, subcutaneous fat distribution, skin thickness, intercostal spaces width, muscle tissue response, and lung positioning. Moreover, The volumes of porcine and humans' lungs are considered comparable, with a total lung capacity of $55 \mathrm{~mL} / \mathrm{kg}$ [27].

Twelve seconds recordings of breath sounds, representing three mechanical inhalation-exhalation cycles, were sampled over the right $7^{\text {th }}$ intercostal space, at the mid-axillary line. This specific site was chosen based on previous studies performed by our group as an appropriate site for auscultation in similar-size animals. Preliminary baseline recordings were sampled and were defined as "volume 0". After this, simple PTX was induced with increasing volumes of $200,400,600,800$, and $1,000 \mathrm{ml}$ of air, injected using a 100 $\mathrm{ml}$ syringe and a $14 \mathrm{G}$ needle into the pleural cavity and underneath the sampling site. Before simulating HTX, the thorax was emptied of air by using the same syringe, until air leak through the needle was absent. Then, an increasing volume of saline ( $0.9 \%$ sodium chloride) was injected in the same position as described above: $200,400,600,800$, and 1,000 ml. Each of the aforementioned states was sampled for a twelve-second recording of breath sounds. The study was conducted in a quiet surrounding, without restrictions on environmental noise during the recordings.

\section{Prototype assimilation}

a. Apparatus assembly: We chose to use two FDA- and CE-approved commercial Thinklabs One digital stethoscopes (Thinklabs Medical LLC, CO, USA) [28], capable of sampling a wide range of frequencies and amplifying them by up to 100 -fold, allowing for recording over a thick chest wall if needed. The stethoscope is round-shaped, with a diameter of $46 \mathrm{~mm}$ and a height of $28 \mathrm{~mm}$, it weighs about 50 grams and is equipped with built-in filters that reduce background noise. The filter we chose was designed to enable a sampling of $20-2,000 \mathrm{~Hz}+/-3 \mathrm{~dB}$. We placed two microphones next to each other, supported by a longitudinal sustaining frame. The microphones were connected to a spatial sampling system assembled at the IDF Medical Corps Medical Engineering Division. The system included a $3.5 \mathrm{~mm}$ adaptor connecting the stethoscopes to a portable myRIO-1900 embedded device (National Instruments, TX, USA) [29]. The sampling apparatus is presented in Figure 2. 
b. Software programming: The sampling algorithm was programmed by the IDF Medical Corps Medical Engineering Division using the LabVIEW platform. The samples recorded by the two stethoscopes were separated into two audio channels for each stethoscope. Therefore, for each recording made, four data sources were obtained. The recordings were analyzed using a MATLAB computing environment and were filtered to omit high-pass and low-pass data points with frequencies of $<60 \mathrm{~Hz}$ and $>2,000 \mathrm{~Hz}$, respectively, that were not originated from the respiratory system. For each recording, the intensity ( ) of the signal was measured in constant gaps of 0.00005 seconds, i.e. 20,000 samples per second. In total, 176 datasets were obtained and were later extracted to an Excel sheet for further data processing and analysis by an external software house (Clinetix - Clinical Kinetics, Tel-Aviv, Israel). The working assumption was that each volume of simple PTX and HTX had a unique acoustic pattern that could be obtained and analyzed, and on which a classification algorithm could be programmed and enable the differentiation of the various volumes of injury compared to the normal state. Due to the small database, we decided not to use a model-based analytical method, such as an artificial neuronal network or a decision tree, which is a better fit for larger quantities of datasets. This led us to use a memory-based method, namely a k-nearest neighbors (KNN) algorithm, which generates prediction by estimating how likely a data point is to be a member of one group or another, depending on what group the data point nearest to it is in. A multi-objective genetic algorithm (MOGA) was used to develop a mathematical detector through the process of artificial evolution. The detector and its features, which are a set of mathematical formulas, were developed by the MOGA as also the KNN algorithm's parameters.

Statistical analysis: Sensitivity, specificity, and accuracy for each injury volume were calculated. Accuracy was defined as

$\mathrm{TP}+\mathrm{TN}$

$\overline{\mathrm{TP}+\mathrm{TN}+\mathrm{FP}+\mathrm{FN}}$

(TP-True positive, TN-True negative, FP-False positive, FN-False negative). In addition to comparing each of the volumes separately from each other, we conducted additional testing combining all observations with comparison to baseline ("volume 0"). A Pearson correlation coefficient was computed to assess the relationship between the real value and the predicted one. Calculations were made using a $\mathrm{C \#}$ programming language in a LINQPad software utility.

Ethics: This study conformed to the Guide for the Care and Use of Laboratory Animals, (National Academy Press, Washington, DC, 1996). Animal care and experimental procedures were approved by the Ethics Committee of The Faculty of Medicine of The Hebrew University of Jerusalem, Israel (approval numbers MD-16-14853-3 and MD-17-15043-3).

Funds: The study was conducted with the help of a research grant from the IDF Medical Corps, and from the Director of the Administration for the Development of Arms and Technological Infrastructure (Maf'at) at the Israeli Ministry of Defense (grant number: 4440838636). 


\section{Results}

Each twelve-seconds recording consisted of 240,000 samples, with a total of 176 datasets obtained. A visual representation of the data was also produced as depicted in Figure 3. The KNN algorithm consisted of a cross-validation process that separated the data into a single testing set and the remaining 175 training sets. Iteratively, we compared each testing set to the complementary training set for a total of 176 different iterations. The algorithm calculated the number of most identical datasets $(K)$ for each testing set. Of the $\mathrm{K}$ results returned, it was the majority decision that determined the success or failure of the prediction. Preliminary examination of the data found that the samples of $200 \mathrm{ml} \mathrm{PTX}$ and HTX were very similar in their acoustic properties to that of a normal state, therefore we decided not to include them in future calculations, in order to improve the algorithm's classification capability.

The algorithm succeeded to predict the presence of PTX and/or HTX at each of the different volumes combined in comparison to baseline, with $81 \%$ sensitivity, $69 \%$ specificity, and $80 \%$ accuracy. The term "combined" refers to the unification of any of the measured volumes of PTX and/or HTX. Thus, addressing it as a single condition without distinguishing between the volumes of injury

When the algorithm was tested to predict the presence of PTX at each of the various volumes combined in comparison to baseline and/or in comparison to the presence of HTX, at each of the various volumes combined, we achieved $63 \%$ sensitivity, $94 \%$ specificity, and $80 \%$ accuracy.

We demonstrated that the algorithm can predict the presence of PTX at each of the various volumes combined in comparison to baseline with $63 \%$ sensitivity, $69 \%$ specificity, and $64 \%$ accuracy. Similar calculations in HTX at each of the various volumes combined in comparison to baseline, achieved $100 \%$ sensitivity, $100 \%$ specificity, and $100 \%$ accuracy.

When we tested the ability of the algorithm to distinguish between the various volumes of PTX, in comparison to baseline, we achieved an average level of sensitivity, specificity, and accuracy of $80 \%$ each.

When the algorithm was tested to predict the presence of HTX at each of the various volumes combined vs. the presence of PTX at each of the various volumes combined, we achieved $100 \%$ sensitivity, $100 \%$ specificity, and $100 \%$ accuracy. Statistically significant positive correlation was found between the real values and the predicted ones for PTX ( $r(72)=0.23, p=0.03), \operatorname{HTX}(r(78)=0.81, p<0.0001)$, and PTX and HTX combined $(r(142)=0.90, p<0.0001)$. Table 1. illustrates each of the above-mentioned measurements.

\section{Discussion}

Technological advances in recent years have resulted in the implementation of artificial intelligence (Al) applications in medicine in general, and in emergency medicine in particular [28-30]. In our study, we introduce an Al-based method capable of accurately identifying air and fluid accumulation in the pleural cavity and assessing their volumes. We have managed to detect the accumulation of $400-1,000 \mathrm{ml}$ of air 
and fluid in the pleural cavity, and distinguish it from a normal state, within twelve-seconds and regardless of background noise, with an accuracy ranging from 64 to 100 percent. When addressing PTX and/or HTX in each one of the various volumes combined, without pointing the exact volume we managed to achieve a sensitivity rate of $81 \%$ alongside an accuracy of $80 \%$ in predicting the condition. As we focused our algorithm in PTX detection and classification without predicting the exact volume of PTX, we obtained a lower sensitivity rate of $63 \%$, but with much higher specificity ( $94 \%$ compared to $69 \%$ in the previous calculation), with $80 \%$ accuracy. When we attempted to classify the exact volume of the injury, in the range of $400 \mathrm{ml}$ to $1,000 \mathrm{ml}$, accuracy dropped to $64 \%$ - a result which was expected, given the relatively small database and the complexity of the calculations. It is important to note that the algorithm fully identified HTX with $100 \%$ sensitivity, $100 \%$ specificity, and $100 \%$ prediction accuracy. However, we were unable to find a good predictor that could predict distinct volumes of HTX. Preliminary examination of the acoustic properties of the HTX samples exhibited a low signal-to-noise ratio, indicating a relatively high rate of background noise in comparison to the level of the desired signal. We believe this was due to the induction of HTX at the same physical location, shortly after draining out the air that was inserted earlier for the purpose of PTX induction. It is possible that some residual air remained in the pleural cavity, accentuating the presence of the injury beyond the increasing volume of saline. Nevertheless, it is important to note that we applied various computational methods, such as the Monte Carlo method, to evaluate the quality and the arbitrariness of the data and found it to be consistently balanced and protected from bias or overfitting effects. This means that the noise or random oscillations in the training set did not impair the predictability ability of the algorithm. This also means that given more data, it is likely that we will be able to significantly increase the prediction accuracy. Although data describing morbidity and mortality of combat casualties exists from early conflicts in the 20th century, most of the modern knowledge and experience are based on US military operations in the Middle East, specifically in Iraq and Afghanistan. It is important to note that in relation to earlier conflicts, modern conflicts are characterized by an increased explosives utilization, such as improvised explosive devices (IEDs), grenades, and shoulder-fired missiles, resulting in higher rates of blast injuries and concomitant blunt thoracic injuries which are difficult and less intuitive to diagnose [30]. Israel Defense Forces (IDF) Medical Corps reviews conducted after Operation Protective Edge in 2014, also suggested tPTX to be one of the most common causes of preventable death on the battlefield, alongside hemorrhage and airway compromise [31]. A population-based study examining PTX incidence in severely traumatized patients in a civilian prehospital setting has shown that up to $20 \%$ of major trauma victims suffered from PTX with up to $15 \%$ of patients receiving emergency chest decompression [32]. Although considerable variation exists in the literature when addressing PTX size assessment, it is considered recommended to treat it when $30 \%-50 \%$ of the hemithorax (600-1,000 ml, respectively), is affected [3335]. Considering the low clinical significance of a $200 \mathrm{ml} \mathrm{PTX}$, and due to the technical issues described above, we decided to exclude those samples from calculations. The samples were recorded in the presence of mild background noise, and although its level was not measured, it can be inferred from the literature as the mean noise level in operating rooms is considered to be around $51-75 \mathrm{~dB}$, as described by Hasfeldt et al. in their review [36]. Due to the limitations of in-vivo human experiments, the porcine model was chosen to demonstrate the feasibility, primarily due to its physiological and anatomical similarity to 
the human thorax. Previous studies described porcine pneumothorax models to be physiologically relevant to human extrapolation [27]. Future experiments should utilize the algorithm in human cadavers to construct human-based datasets.

A genetic algorithm was used to determine various parameters consisting the $\mathrm{KNN}$ algorithm and was also utilized in the process of creating the mathematical detector which identified the unique differentiating signature for each volume. Genetic algorithms are a family of optimization algorithms designed to streamline problem-solving by artificially activating a process that simulates the natural selection process underpinning the theory of evolution [37]. The method is based on applying small changes ("mutations") to randomly selected solutions for a certain problem, while producing new solutions and selecting those who better fit the target that has been defined by a "fitness" function and its stopping criteria until reaching a near-perfect optimization within several runs ("generations"). This method is best used for solving problems for which the target is known but the way to attain it is unclear.

Among the contemporary technological solutions for prehospital thoracic trauma detection, the most widespread modality is emergency ultrasonography. The E-FAST protocol may assist in assessing the presence of PTX and HTX, and to a lesser extent, its severity. Although the majority of studies present a very high specificity (>95\%) for the diagnosis of PTX in patients with thoracic trauma, they also exhibit a low sensitivity ( 50\%) when compared to other imaging modalities [38]. Prehospital emergency ultrasonography is noninvasive, portable, and inexpensive. Moreover, it has the advantage of being functional even in loud surroundings.

Nevertheless, it is heavily relied on provider experience and therefore may be time consuming, and it cannot differ acute HTX from chronic pleural effusions [39]. Although the latter may provide a challenge for our modality as well, we offer a non-provider-dependent rapid and objective detection modality, which may provide the actual volume of injury with higher sensitivity rate.

Diagnosing PTX using various acoustic techniques has previously been demonstrated in the literature. In their exploratory study, Mansy et al. demonstrated how the presence of PTX affects the transmission of sound waves across the thorax. During the study, which was conducted on 19 patients with iatrogenic PTX. Although the authors suggest a potential method for distinguishing between baseline and the presence of PTX, they present an invasive method [38]. Peng et al. presented a computational model simulating acoustic transmission from the lung to the thoracic surface in a porcine model under PTX. They found that introducing sound waves via the endotracheal tube may be measured by the transmitted waves from the thorax by a laser Doppler vibrometer [39]. Thus, presenting a potential method for detecting pulmonary abnormalities, including PTX. A canine model for distinguishing PTX from baseline using vibrational response on the thorax following an external acoustic stimulation introduced via the endotracheal tube was described by Royston et al. [40]. Early studies describing the acoustic characteristics of breath sound suggested PTX cause amplitude reduction, decrease in high-frequency components, and reduced amplitude variation [41]. Hayashi introduced an external device that analyzed and converted the frequency of thoracic auscultatory sounds to numerical values and demonstrated how 
the presence of PTX could be detected with a sensitivity and specificity of $71.4 \%$ and $100 \%$, respectively. His results were based on ten breath cycle recordings, which are equivalent to about 30-40 seconds. Confirmatory diagnosis of PTX was made by chest x-ray or a chest tomography and no data regarding the volume of the injury was provided [42].

In view of current literature, our method presents higher sensitivity and accuracy with shorter measurement times, regardless of background noise, and distinguishing PTX and HTX as small as 400 $\mathrm{ml}$ in volume. Moreover, our method utilized auscultatory data from a single spot on the thorax with no need for external excitation of any kind. Furthermore, while most studies refer to PTX diagnosis only, we managed to identify the presence of injected fluid in the pleural cavity, simulating HTX. Another clinical implication of our modality is that in the case of HTX, optimal resuscitation could be applied, and additional medication could be administered, such as tranexamic acid or fresh frozen plasma infusion. Moreover, chest tube positioning might be different if prior knowledge is available. In the case of PTX, one should point the tip of the tube upwards, while in the case of HTX, it should be pointed posteriorly and caudally. Due to rapid application of our modality, a near-instant follow-up could be applied, and patients treated with chest tubes could be monitored regardless of background noise. Equally important, proper use of our device can prevent unnecessary treatment in patients who do not require chest drainage.

\section{Limitations}

For PTX and HTX simulation, we induced an isolated injury while in the real-world, there may be a combined injury resulting in hemopneumothorax and concomitant pulmonary contusions that may potentially affect the results of auscultation. Moreover, natural breathing was absent due to the ventilated animal model and the absent of normal blood flow and pulse may also have influenced the acoustic properties of the respiratory sounds. Another limitation was the presence of background noise, the level of which we did not measure. Thus, it is possible that the background noise may have caused artifacts in the signals detected. Nevertheless, the stethoscopes were designed to pick up signals coming from directly in front of them only; so that the potential interruption of background noise was minimal if existing at all. In addition, we applied two levels of filtering, the first was the built-in filter in the stethoscope and the second was the frequencies filter we built as part of the sampling algorithm. As our modality is designated to be utilized in field conditions, and on the battlefield, future studied would address that point and test our algorithm's ability to sample and classify the signals even when applied in loud environments, of up to $120 \mathrm{~dB}$. Next, although we sampled a high number of measurements per second, the study comprised of only four animal models. Thus, selection bias and overfitting in the prediction algorithm should be taken into consideration. Moreover, the evaluation process was conducted through cross-validation. Although it was the best that was available for our datasets, it is not as reliable as testing the method on an entirely independent sample, which was not in the scope of the current experiment. Nevertheless, several preliminary tests were performed to ensure the quality of the data despite the small amount of data. A Monte Carlo simulation method for estimating the arbitrariness of 
the correlations detected in the recorded samples showed that the recorded data was balanced, not arbitrary, and protected from bias or overfitting effects. Confirmation of recording above the area of the injury was made retrospectively, after drainage of air/saline from the intervention point on the thorax. No definitive measures, such as chest x-ray or thoracoscopy were used for injury location proof. Therefore, we cannot say with absolute certainty whether the signal measurement was performed precisely above the injury. However, in the real-world, auscultation will be done over an arbitrary spot on the patient's hemithorax with no prior knowledge of the location of the injury, and we expect a diagnosis device could indicate the presence of PTX or HTX even if not placed directly above them.

\section{Conclusions}

We have managed to detect and classify an abnormal accumulation of air and fluid in the pleural cavity in a porcine model simulating hemopneumothorax by utilizing a prediction algorithm which was optimized by an artificial evolution method. This novel method has the potential to non-invasively detect and quantify within seconds the presence of hemopneumothorax, independently of external factors that may delay diagnosis and treatment. Although promising, it seems that our modality can better distinguish fluid accumulation rather than air, most probably secondary to methodical issues, as thoroughly discussed. Given a larger dataset, favorable results in high resolution detection of air accumulation will follow. Beyond the potential use in field conditions and on the battlefield, our method may also present potential benefits for use in trauma bays, intensive care units, catheterization rooms, and operating rooms, monitoring for complications. Further studies are required to improve the algorithm's predictability rate and to embed it into a portable prototype.

\section{List Of Abbreviations}

tPTX - Tension pneumothorax

PTX - Pneumothorax

HTX - Hemothorax

E-FAST - Extended Focused Assessment with Sonography for Trauma

Al - Artificial intelligence

$\mathrm{KNN}$ - k-nearest neighbors

MOGA - Multi-objective genetic algorithm

IED - Improvised explosive devices

\section{Declarations}


Ethics approval and consent to participate: This study conformed to the Guide for the Care and Use of Laboratory Animals, (National Academy Press, Washington, DC, 1996). Animal care and experimental procedures were approved by the Ethics Committee of The Faculty of Medicine of The Hebrew University of Jerusalem, Israel (approval numbers MD-16-14853-3 and MD-17-15043-3).

Consent for publication: Not applicable

Availability of data and materials: The datasets generated and/or analysed during the current study are not publicly available due to intellectual property protection but are available from the corresponding author on reasonable request.

Competing interests: The authors declare that they have no competing interests

Funding: The research was supported by the Israel defense forces (IDF) Medical Corps and Directorate of Defense Research \& Development, Israeli Ministry of Defense (IMOD DDR\&D).

Authors' contributions: AS has contributed to the conception, design of work, acquisition, analysis, and interpretation of data, and drafted the manuscript; NM has contributed to the acquisition, analysis, and interpretation of data, RG has contributed to the acquisition, analysis, and interpretation of data, LWA has contributed to the design of work and substantively revised it, RFM has contributed to the acquisition, analysis, and interpretation of data, AE has substantively revised it, DN has contributed to the design of work and substantively revised it.

Acknowledgements: The authors would like to thank Anat Lyubin and Eran Shasha for their contribution in programing the sampling algorithm.

\section{References}

1. Eastridge BJ, Mabry RL, Seguin P, Cantrell J, Tops T, Uribe P, et al. Death on the battlefield (20012011): implications for the future of combat casualty care. J Trauma Acute Care Surg. United States; 2012;73:S431-7. 
2. Butler FK. TCCC Updates: Two Decades of Saving Lives on the Battlefield: Tactical Combat Casualty Care Turns 20. J. Spec. Oper. Med. a peer Rev. J. SOF Med. Prof. United States; 2017. p. 166-72.

3. Butler FKJ, Blackbourne LH. Battlefield trauma care then and now: a decade of Tactical Combat Casualty Care. J Trauma Acute Care Surg. United States; 2012;73:S395-402.

4. McPherson JJ, Feigin DS, Bellamy RF. Prevalence of tension pneumothorax in fatally wounded combat casualties. J Trauma. United States; 2006;60:573-8.

5. Kelly JF, Ritenour AE, Mclaughlin DF, Bagg KA, Apodaca AN, Mallak CT, et al. Injury Severity and Causes of Death From Operation Iraqi Freedom and Operation Enduring Freedom: 2003 - 2004 Versus 2006. 2006;64:11-5.

6. Butler F.K., Kotwal R.S. (2017) Tactical Combat Casualty Care. In: Martin, M., Beekley, A., Eckert M. (eds) Front Line Surgery. Springer, Cham.

7. Holcomb JB, McMullin NR, Pearse L, Caruso J, Wade CE, Oetjen-Gerdes L, et al. Causes of death in U.S. Special Operations Forces in the global war on terrorism: 2001-2004. Ann Surg. United States; 2007;245:986-91.

8. Keneally RJ, Shields $\mathrm{CH}$, Hsu A, Prior HI, Creamer KM. Pediatric Thoracic Trauma in Iraq and Afghanistan. Mil Med. England; 2018;183:e596-602.

9. Tien HC, Jung V, Rizoli SB, Acharya S V, MacDonald JC. An evaluation of tactical combat casualty care interventions in a combat environment. J Am Coll Surg. United States; 2008;207:174-8.

10. Winkle JM \& Legome, E. Initial evaluation and management of penetrating thoracic trauma in adults. In: UpToDate, Post, TW (Ed), UpToDate, Waltham, MA, 2014.

11. Legome, E. Initial evaluation and management of blunt thoracic trauma in adults. In: UpToDate, Post, TW (Ed), UpToDate, Waltham, MA, 2014.

12. Light RW \& Lee YCG. Clinical presentation and diagnosis of pneumothorax. In: UpToDate, Post, TW (Ed), UpToDate, Waltham, MA, 2014.

13. Ianniello S, Piccolo CL, Trinci M, Ajmone Cat CA, Miele V. Extended-FAST plus MDCT in pneumothorax diagnosis of major trauma: time to revisit ATLS imaging approach? J Ultrasound. 2019;22:461-9.

14. Ianniello S, Di Giacomo V, Sessa B, Miele V. First-line sonographic diagnosis of pneumothorax in major trauma: accuracy of e-FAST and comparison with multidetector computed tomography. Radiol Med. Italy; 2014;119:674-80.

15. Bokhari F, Brakenridge S, Nagy K, Roberts R, Smith R, Joseph K, et al. Prospective evaluation of the sensitivity of physical examination in chest trauma. J Trauma. United States; 2002;53:1135-8.

16. Kong VY, Sartorius B, Clarke DL. The accuracy of physical examination in identifying significant pathologies in penetrating thoracic trauma. Eur $\mathrm{J}$ trauma Emerg Surg Off Publ Eur Trauma Soc. Germany; 2015;41:647-50.

17. Chen SC, Markmann JF, Kauder DR, Schwab CW. Hemopneumothorax missed by auscultation in penetrating chest injury. J Trauma. United States; 1997;42:86-9. 
18. Chen SC, Chang KJ, Hsu CY. Accuracy of auscultation in the detection of haemopneumothorax. Eur J Surg. England; 1998;164:643-5.

19. Paul Mayo MD, Thoracic Ultrasound: Indications, Advantages and Technique. In: UpToDate, Post TW (Ed), UpToDate, Waltham, MA. (Accessed on August 06, 2019.).

20. Zhang M, Liu Z-H, Yang J-X, Gan J-X, Xu S-W, You X-D, et al. Rapid detection of pneumothorax by ultrasonography in patients with multiple trauma. Crit Care. 2006;10:R112.

21. Staub LJ, Biscaro RRM, Kaszubowski E, Maurici R. Chest ultrasonography for the emergency diagnosis of traumatic pneumothorax and haemothorax: A systematic review and meta-analysis. Injury. Netherlands; 2018;49:457-66.

22. Levy PD, Wielinski T, Greszler A. Micropower impulse radar: a novel technology for rapid, real-time detection of pneumothorax. Emerg Med Int. Egypt; 2011;2011:279508.

23. Hocagil H, Hocagil AC, Karacabey S, Akkaya T, Simsek G, Sanri E. A prospective study: Is handheld micropower impulse radar technology (Pneumoscan) a promising method to detect pneumothorax? Ulus Travma Acil Cerrahi Derg. Turkey; 2015;21:344-51.

24. Rehfeldt M, Slagman A, Leidel BA, Möckel M, Lindner T. Point-of-Care Diagnostic Device for Traumatic Pneumothorax: Low Sensitivity of the Unblinded PneumoScan ${ }^{\mathrm{TM}}$. Emerg Med Int. 2018;2018:1-6.

25. Rich PB, Dulabon GR, Douillet CD, Listwa TM, Robinson WP, Zarzaur BL, et al. Infrared thermography: a rapid, portable, and accurate technique to detect experimental pneumothorax. J Surg Res. United States; 2004;120:163-70.

26. Fast Detection of a Punctured Lung. Science and Technology Review. Retrieved from https://str.IInl.gov/str/Oct07/Chang.html. (2007, October 11).

27. Oveland NP, Sloth E, Andersen G, Lossius HM. A porcine pneumothorax model for teaching ultrasound diagnostics. Acad Emerg Med Off J Soc Acad Emerg Med. 2012;19:586-92.

28. Thinklabs One Digital Stethoscope. https://www.thinklabs.com/one-digital-stethoscope. Accesed June 30, 2019.

29. myRIO Student Embedded Device. http://www.ni.com/en-il/shop/select/myrio-student-embeddeddevice. Accesed June 30, 2019.

30. Zong Z-W, Wang Z-N, Chen S-X, Qin H, Zhang L-Y, Shen Y, et al. Chinese expert consensus on echelons treatment of thoracic injury in modern warfare. Mil Med Res. England; 2018;5:34.

31. Avi B, Elon G, Baruch EN, Avi S, Gilad T, Moran L, et al. Augmentation of point of injury care: Reducing battlefield mortality-The IDF experience. Injury [Internet]. 2015/11/18. Netherlands; 2016;47:9931000. Available from: https://www.ncbi.nlm.nih.gov/pubmed/26725147

32. Di Bartolomeo S, Sanson G, Nardi G, Scian F, Michelutto V, Lattuada L. A population-based study on pneumothorax in severely traumatized patients. J Trauma. United States; 2001;51:677-82.

33. RW Light. Clinical presentation and diagnosis of pneumothorax. Post TW, ed. UpToDate. Waltham, MA: UpToDate Inc. https://www.uptodate.com (Accessed on October 21, 2019.). 
34. RW Light. Treatment of secondary spontaneous pneumothorax in adults. Post TW, ed. UpToDate. Waltham, MA: UpToDate Inc. https://www.uptodate.com (Accessed on October 21, 2019.).

35. RW Light. Treatment of primary spontaneous pneumothorax in adults. Post TW, ed. UpToDate. Waltham, MA: UpToDate Inc. https://www.uptodate.com (Accessed on October 21, 2019.).

36. Hasfeldt D, Laerkner E, Birkelund R. Noise in the operating room-what do we know? A review of the literature. J perianesthesia Nurs Off J Am Soc PeriAnesthesia Nurses. United States; 2010;25:380-6.

37. Goldberg D. Genetic Algorithms in Search, Optimization, and Machine Learning. Boston, MA: Addison-Wesley Professional; 1989.

38. Mansy HA, Balk RA, Warren WH, Royston TJ, Dai Z, Peng Y, et al. Pneumothorax effects on pulmonary acoustic transmission. J Appl Physiol. 2015;119:250-7.

39. Peng Y, Dai Z, Mansy HA, Henry BM, Sandler RH, Balk RA, et al. Sound transmission in porcine thorax through airway insonification. Med Biol Eng Comput. 2016;54:675-89.

40. Royston TJ, Zhang X, Mansy HA, Sandler RH. Modeling sound transmission through the pulmonary system and chest with application to diagnosis of a collapsed lung. J Acoust Soc Am. United States; 2002;111:1931-46.

41. Mansy HA, Royston TJ, Balk RA, Sandler RH. Pneumothorax detection using computerised analysis of breath sounds. Med Biol Eng Comput. United States; 2002;40:526-32.

42. Hayashi N. Detection of pneumothorax visualized by computer analysis of bilateral respiratory sounds. Yonago Acta Med. Japan; 2011;54:75-82.

\section{Tables}

Due to technical limitations, Table 1 is only available as a download in the Supplemental Files section.

\section{Figures}




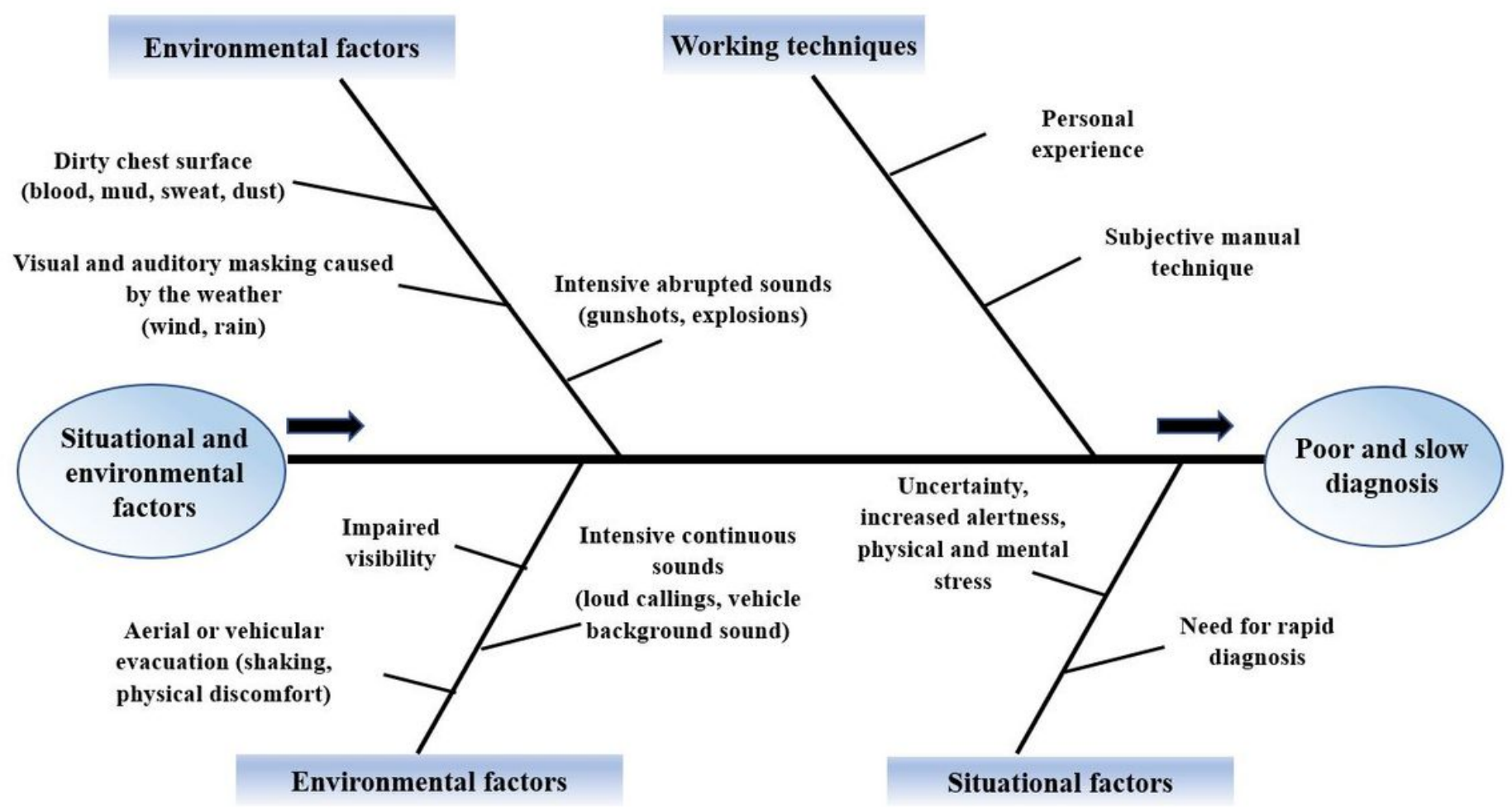

\section{Figure 1}

A cause and effect diagram detailing the environmental and situational factors that may impair clinical judgment and disturb physical examination on the battlefield.
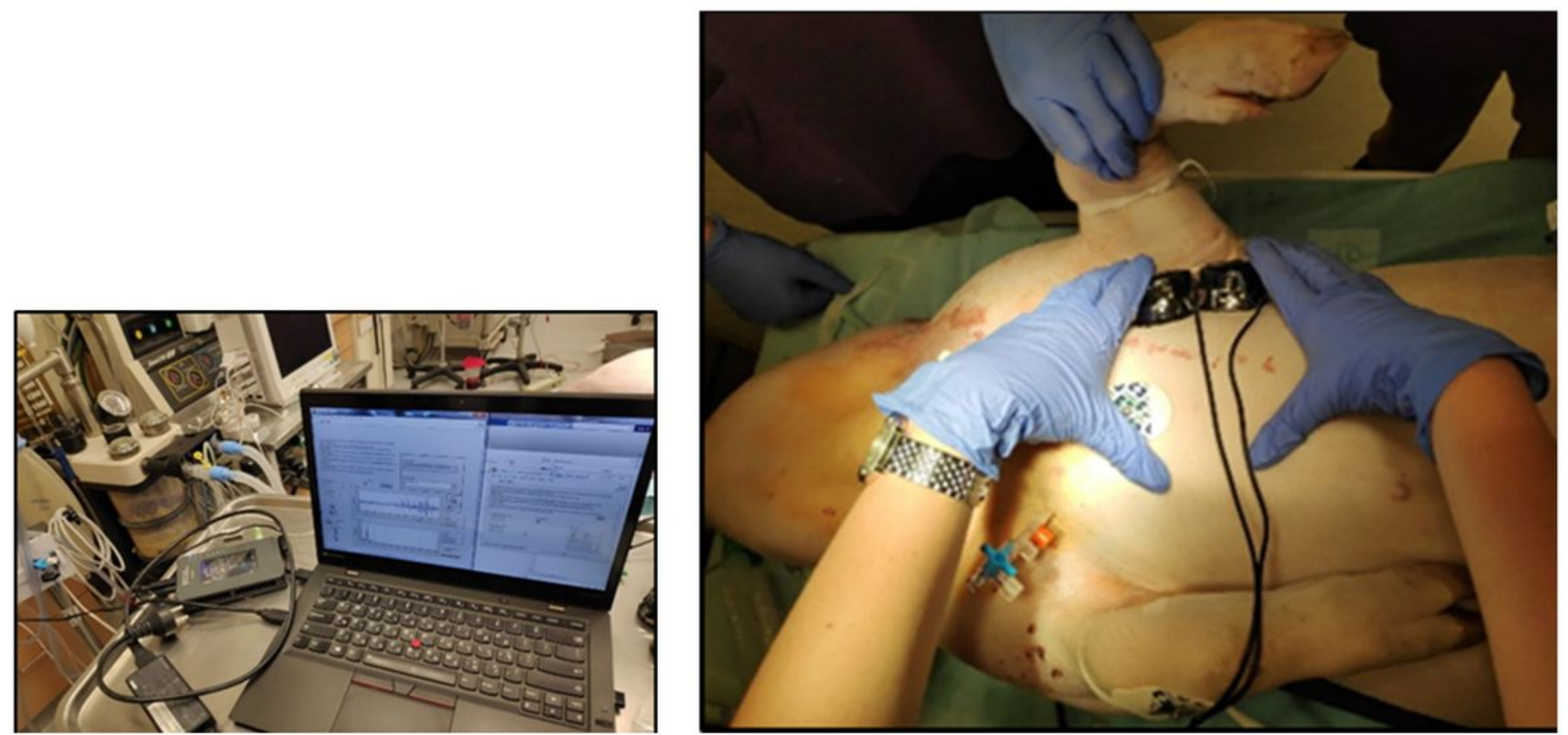

Figure 2 
The recording apparatus, consisting of two digital stethoscopes connected to the sampling card. Apparatus' approximate dimensions: Length: ten centimeters; Width: five centimeters; Height: three centimeters; Weight: 110 grams.

\begin{tabular}{|c|c|c|c|c|c|c|}
\hline $\mathrm{vol}=0$ & vol $=\mathbf{2 0 0}$ & $\mathrm{vol}=400$ & $\mathrm{vol}=600$ & vol $=800$ & vol $=1000$ & time \\
\hline-0.003147765 & 0.00527999 & -0.005280791 & 0.001282211 & 0.004940968 & 0.000057328 & 0.00005 \\
\hline-0.00386862 & 0.010674365 & -0.00546393 & -0.003228215 & 0.008912077 & 0.000057328 & 0.0001 \\
\hline-0.004535408 & 0.015915245 & -0.005574587 & -0.007687208 & 0.01277273 & 0.000057328 & 0.00015 \\
\hline-0.005138628 & 0.020934398 & -0.005609785 & -0.012037042 & 0.016472957 & 0.000057328 & 0.0002 \\
\hline-0.005669726 & 0.025666969 & -0.005567618 & -0.016221648 & 0.019965008 & 0.000057328 & 0.00025 \\
\hline-0.006121227 & 0.030052391 & -0.005447304 & -0.020187422 & 0.023204024 & 0.000057328 & 0.0003 \\
\hline-0.006486846 & 0.034035231 & -0.005249207 & -0.02388397 & 0.026148677 & 0.000057328 & 0.00035 \\
\hline-0.006761593 & 0.037565961 & -0.004974859 & -0.027264828 & 0.028761736 & 0.000057328 & 0.0004 \\
\hline-0.006941843 & 0.040601624 & -0.004626945 & -0.030288097 & 0.031010593 & 0.000057328 & 0.00045 \\
\hline-0.00702539 & 0.043106403 & -0.004209288 & -0.032917023 & 0.032867704 & 0.000057328 & 0.0005 \\
\hline-0.007011484 & 0.045052081 & -0.003726804 & -0.035120491 & 0.034310955 & 0.000057328 & 0.00055 \\
\hline-0.006900833 & 0.046418382 & -0.003185438 & -0.036873436 & 0.035323962 & 0.000057328 & 0.0006 \\
\hline-0.006695596 & 0.047193181 & -0.0025921 & -0.038157155 & 0.035896261 & 0.000057328 & 0.00065 \\
\hline-0.006399338 & 0.047372607 & -0.00195456 & -0.038959528 & 0.036023429 & 0.000057328 & 0.0007 \\
\hline-0.006016975 & 0.046961008 & -0.001281348 & -0.039275135 & 0.035707111 & 0.000057328 & 0.00075 \\
\hline-0.00555469 & 0.045970797 & -0.000581641 & -0.039105278 & 0.03495495 & 0.000057328 & 0.0008 \\
\hline-0.005019834 & 0.04442218 & 0.000134881 & -0.038457894 & 0.033780437 & 0.000057328 & 0.00085 \\
\hline-0.004420803 & 0.042342775 & 0.000858163 & -0.037347371 & 0.032202676 & 57328 & 0.0009 \\
\hline-0.003766907 & 0.03976711 & 7928 & -0.035794276 & 0.03024606 & 57328 & 0.00095 \\
\hline-0.003068216 & 0.036736034 & 0.002283829 & -0.033824991 & 0.027939881 & 0.000057328 & 0.001 \\
\hline-0.0023354 & 0.033296046 & 0.0029656 & -0.031471256 & 0.02531787 & 0.000057328 & 0.00105 \\
\hline-0.001579559 & 0.029498537 & 0.003613215 & -0.028769647 & 0.022417662 & 0.000057328 & 0.0011 \\
\hline-0.000812048 & 0.025398972 & 0.004217039 & -0.025760982 & 0.019280232 & 0.000057328 & 0.00115 \\
\hline$-4.42906 \mathrm{E}-05$ & 0.021056029 & 0.004767972 & -0.022489675 & 0.015949266 & 0.000057328 & 0.0012 \\
\hline 0.00071239 & 0.016530697 & 0.005257601 & -0.019003037 & 0.012470516 & 0.000057328 & 0.00125 \\
\hline 0.001446959 & 0.011885358 & 0.00567832 & -0.015350548 & 0.008891111 & 0.000057328 & 0.0013 \\
\hline 0.002148831 & 0.007182856 & 0.00602346 & -0.011583103 & 0.005258881 & 0.000057328 & 0.00135 \\
\hline 0.00280804 & 0.002485584 & 0.00628739 & -0.007752248 & 0.001621657 & 0.000057328 & 0.0014 \\
\hline 0.00341539 & -0.002145405 & 0.006465611 & -0.003909422 & -0.001973407 & 0.000057328 & 0.00145 \\
\hline 0.00396259 & -0.006651265 & 0.006554837 & -0.000105201 & -0.005480493 & 0.000057328 & 0.0015 \\
\hline
\end{tabular}

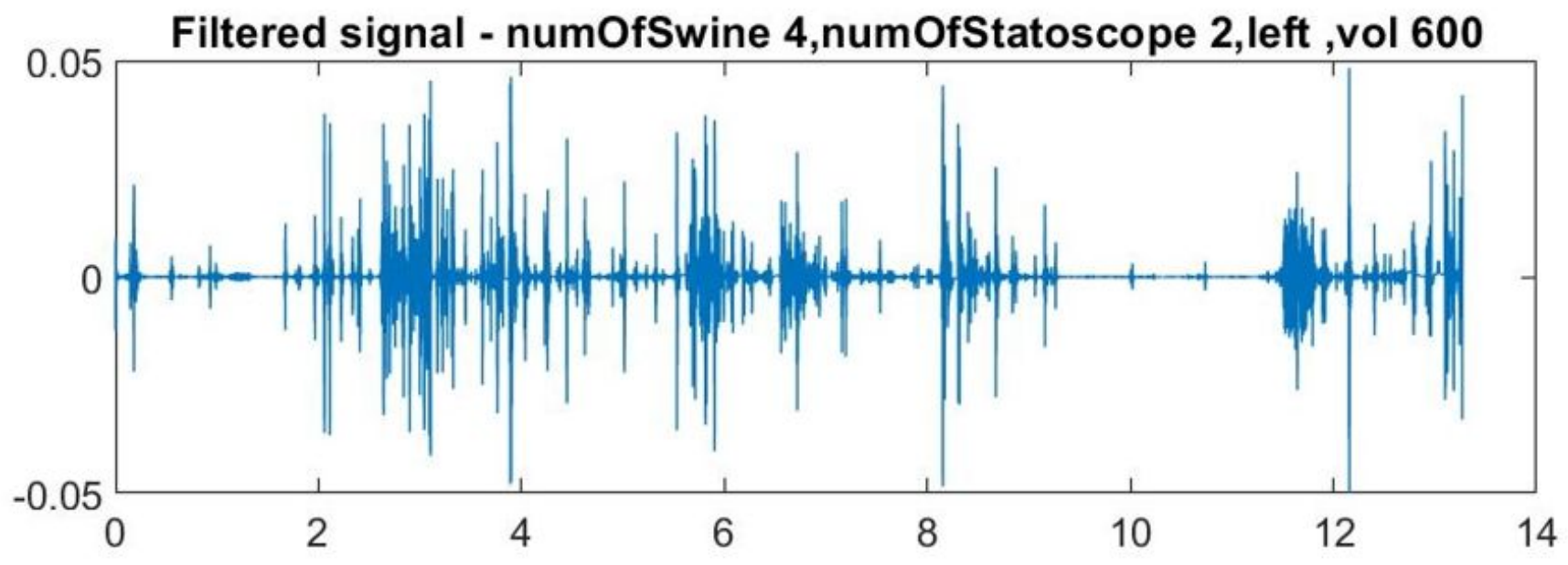

Figure 3

An example of the time-series data. The upper image represents the tabular time-series data. The lower image represents a visual representation of the time-series. Horizontal axis: time series; vertical axis: 
intensity.

\section{Supplementary Files}

This is a list of supplementary files associated with this preprint. Click to download.

- Table1.jpg 